\title{
Solidão e Depressão: Relações com Características Pessoais e Hábitos de Vida em Universitários
}

\author{
Sabrina Martins Barroso* (D), Nadyara Regina de Oliveira, \& Valéria Sousa de Andrade \\ Universidade Federal do Triângulo Mineiro, Uberaba, MG, Brasil
}

\begin{abstract}
RESUMO - Este trabalho avaliou a solidão e a depressão em universitários, verificando sua relação com hábitos, características do curso e suporte social. Participaram 574 universitários. Utilizou-se a Escala de Solidão UCLA-BR, Escala de Suporte Social, Questionário sobre a Saúde do Paciente e questionário complementar. Houve triagem para depressão em $38,7 \%$ dos estudantes e $53 \%$ sentia solidão mínima. A maioria dos estudantes fazia quatro ou mais refeições, dormia entre 6 e 8 horas, considerava ter amigos e lazer. Não fumavam, mas bebiam. Houve correlação entre solidão e depressão $($ rho $=0,42)$ e dessas condições com hábitos dos universitários, área do curso e suporte social. Conclui-se que o estado emocional de universitários avaliados é preocupante e intervenções são necessárias.
\end{abstract}

PALAVRAS-CHAVE: solidão, depressão, suporte social, universitários, UCLA-BR

\section{Loneliness and Depression: Relations with Personal Characteristics and Life Habits in University Students}

\begin{abstract}
This study evaluated loneliness and depression of college students to verify its relationship with their habits, course characteristics, and social support. It was used the UCLA-BR Loneliness Scale, Social Support Scale, Patient Health Questionnaire, and a complementary questionnaire to evaluate 574 students. Depression was screened in $38.7 \%$ of the students, and 53\% of them showed minimal loneliness. Most students had four or more daily meals, slept 6 to 8 hours, considered having friends and leisure, did not smoke, but usually drank. There was a correlation between loneliness and depression $($ rho $=0.42)$ and of these with the students' habits, course area, and social support. It was concluded that the emotional state of the students is worrying and that interventions are necessary.
\end{abstract}

KEYWORDS: loneliness, depression, social support, undergraduate students, UCLA-BR

A depressão é um transtorno multifatorial que pode surgir em qualquer período da vida e acarreta grande custo pessoal e social, aumentando o risco para comportamentos autodestrutivos, podendo, em último caso, levar ao suicídio. Segundo a Organização Pan-Americana de Saúde (OPS, 2018) esse transtorno atinge cerca de 300 milhões de pessoas e é a principal causa de incapacidade em todo mundo, sendo também considerado o segundo maior problema mundial de saúde pública (Marcus, van Ommeren, Chisholm, \& Saxena, 2012; World Health Organization [WHO], 2012). Exatamente pelas graves consequências que acarreta, estudos de rastreio são importantes para a prevenção do surgimento do transtorno depressivo (Aquino, Cardoso, \& Pinho, 2019).

No Brasil, a estimativa oficial da prevalência da depressão na população acima de 18 anos é de 7,6\% (Instituto Brasileiro de Geografia e Estatística [IBGE], 2014), sendo que a faixa etária de 18 a 29 anos corresponde a 3,9\% desse total. Kessler et al. (2010) avaliaram a prevalência da depressão em 18 países estimaram a prevalência pontual para depressão no Brasil em 10,4\% e a prevalência ao longo da vida do país em 18,4\%. Molina et al. (2012) atentam que esse número pode estar minimizado pelo subdiagnóstico, dada a falta de preocupação observada na atenção com os problemas de saúde mental.

$\overline{\text { Apoio: FAPEMIG }}$

* E-mail: smb.uftm@gmail.com

- Submetido: 27/12/2016; Revisado: 02/10/2017; Aceito: 24/02/2018 
A faixa etária dos 18 aos 29 anos é a que se encontra a maior parte dos estudantes universitários das instituições federais brasileiras (Fórum Nacional de Pró-reitores de Assuntos Comunitários e Estudantis [FONAPRACE], 2019). Esses estudantes, jovens adultos, se encontram numa fase marcada pela tomada de decisões significativas, por diversas mudanças e pressões sociais (Souza, 2017). Esse período da vida é considerado o de maior risco para as primeiras manifestações comportamentais de perturbações mentais graves, que são suscetíveis a gatilhos emocionais, como o estresse aumentado (Silveira, Brandão, \& Roma-Torres, 2011). Para além das demandas naturais da faixa etária, as exigidas pelo contexto da universidade podem agravar problemas de saúde mental pré-existentes ou aumentar sua probabilidade de seu surgimento (Bolsoni-Silva \& Guerra, 2014; Rondina, Piovezzani, Oliveira, \& Martins, 2018; Silveira et al., 2011).

Os estudos sobre a saúde mental universitária têm ganhado foco recentemente (Bardagi \& Hutz, 2011; Carlotto, 2013), mas eles ainda têm privilegiado bastante a área da saúde. A prevalência da depressão dos universitários varia bastante entre os estudos. Em Santa Catarina, Sakae, Padão e Jornada (2010), realizaram um estudo com estudantes de diferentes cursos da área das Ciências da Saúde, os estudantes de Psicologia foram os que apresentam prevalência mais alta de depressão (13,3\%), ficando os de Educação Física com a menor triagem para o transtorno $(3,1 \%)$. Enquanto Cáceres, Cascaes e Büchele (2010) observaram a prevalência de depressão de 5,67\% entre estudantes de Enfermagem. Ainda sobre os estudantes de enfermagem, Furegato, Santos e Silva (2010) observaram 15,4\% de depressão entre os bacharéis e $28,6 \%$ na licenciatura. No curso de Medicina, Paula et al. (2014) avaliaram estudantes do Ceará e observaram depressão em 28,8\%, enquanto, no estado de São Paulo, Serra, Dinato, Mattos e Caseiro (2015) observaram 30\% de prevalência de depressão entre os acadêmicos e em Santa Catarina, Tabalipa et al. (2015) observaram 32,8\% de triagem de depressão entre os estudantes.

A solidão é um fator de risco para a depressão, ideação e comportamento suicida, bem como para uma variedade de outros resultados psicológicos e fisiológicos negativos (Hackett, Hamer, Endrighi, Brydon, \& Steptoe, 2012; Oliveira \& Silva, 2014) e déficits cognitivos e declínio cognitivo acelerado (Ellwardt et al., 2013; Tyler et al., 2011). Tyler et al. (2011) definem solidão como uma condição psicológica debilitante, caracterizada por um profundo sentimento de isolamento social, vazio e ameaça pessoal. Outros autores indicam que a solidão consiste em um sentimento angustiante e desagradável, que acompanha a percepção de que as necessidades sociais não são atendidas pela quantidade ou qualidade dos relacionamentos existentes (Ellwardt et al., 2013). Ela parece se manifestar de forma distinta em cada fase do desenvolvimento humano e possui dois componentes, um cognitivo e um emocional (Liepins \& Cline, 2011). Portanto, não deriva de estar objetivamente sozinho. Apenas nos casos onde há sentimentos negativos existe solidão, podendo ocorrer mesmo quando um indivíduo está em um ambiente com outras pessoas (Liepins \& Cline, 2011; Russell et al., 2012).

A solidão começa a surgir em alguns estudos sobre a população universitária. Rhodes (2014) indicou que os estudantes se sentiam solitários, em especial no primeiro ano da faculdade e atribuíam sua solidão ao novo ambiente, a lidar de forma ineficiente com situações estressantes e possuir sentimentos de insatisfação nos relacionamentos. O autor também observou que os estudantes mais solitários apresentavam maior presença de problemas físicos e mentais, mostrando o impacto negativo da solidão nessa população. Em contexto nacional o estudo do FONAPRACE (2019) indicou que $83,5 \%$ dos estudantes vivenciavam alguma dificuldade emocional que interferia em sua vida acadêmica e $23,7 \%$ indicou que seus problemas emocionais impactavam seu desempenho acadêmico. Esse estudo mostrou, ainda, que $23,5 \%$ dos universitários disseram que uma das suas dificuldades emocionais era o sentimento de solidão. Souza (2017), também conduziu um estudo em que observou que o sentimento de solidão esteve presente em $47 \%$ dos estudantes investigados.

Outros aspectos que podem influenciar a saúde emocional dos universitários são o suporte social e seus hábitos cotidianos. $\mathrm{O}$ suporte social pode ser definido como um processo interativo, no qual a pessoa pode conseguir ajuda de ordem emocional, instrumental ou financeira (Siqueira, 2008). Ou seja, é uma percepção subjetiva de ter alguém com quem contar em momentos de necessidade. Estudos anteriores observaram a relação entre solidão e falta de suporte social (Ellwardt et al., 2013; Oliveira \& Silva, 2014; Rhodes, 2014; Russell et al., 2012). Tais estudos têm observado que pessoas que se percebem amadas e protegidas não se sentem solitárias, mesmo que estejam distantes de seus familiares e amigos (Rhodes, 2014; Russell et al., 2012).

Esses fatores se somam a outros para aumentar o risco de sofrimento emocional nos estudantes. Estudos sobre condições emocionais indicaram que ser mulher representa um fator de risco para transtornos de humor (OPS, 2018; Kessler et al., 2010; WHO, 2012). No estudo de Hackett et al. (2012) as pessoas mais solitárias mostraram pior saúde física e as mulheres mais solitárias apresentaram mais estresse, maior índice de massa corporal e fumavam mais do que as mulheres menos solitárias e do que os homens. Costa, Nakata e Morais (2015) mostraram, ainda, a importância de entender os hábitos de lazer e a presença de crenças religiosas ao investigar o estado emocional de adultos. Enquanto Baldissera e Bueno (2012) ressaltaram a relevância do exercício físico como estratégia para promoção do bem-estar.

Considerando os possíveis impactos da depressão e da solidão em universitários, o presente estudo avaliou a intensidade da solidão e realizou a triagem para depressão nos estudantes de uma universidade do interior de Minas Gerais, verificando a relação entre esses aspectos e os hábitos de vida, características dos cursos de graduação e nível de suporte social dos universitários. 


\section{MÉTODO}

\section{Participantes}

Participaram do estudo 574 estudantes de graduação da Universidade Federal do Triângulo Mineiro (UFTM), com idade entre 18 e 54 anos ( $=22,27$; D. P. $+4,72)$. Desses, $61,1 \%$ eram mulheres e $93,4 \%$ solteiros. Houve representantes de 22 dos 25 cursos oferecidos pela instituição, sendo 103 estudantes (18\%) de cursos das Ciências Humanas, 206 (36\%) das Ciências da Saúde, 186 $(32,5 \%)$ das Ciências Exatas e 78 (13,6\%) das Ciências Naturais.

Quanto à renda familiar, 34,6\% declararam renda de $3 \mathrm{a} 4$ salários mínimos, $25,2 \%$ renda de 5 a 7 salários, mas $86,4 \%$ declararam não ter emprego remunerado. As informações de caracterização da amostra e hábitos encontram-se apresentadas na Tabela 1 dos resultados.

\section{Instrumentos}

Questionário sobre a Saúde do Paciente (PHQ-9). Validada para o Brasil (Santos et al., 2013), faz a triagem para Episódio Depressivo Maior (EDM) por meio de nove afirmações baseadas no Manual Diagnóstico e Estatístico de Transtornos e alternativas de resposta dadas em escala likert de quatro pontos. O PHQ-9 mostrou validade e fidedignidade (alfa de Cronbach $=0,89$ ). Segundo ponto de corte de Santos et al. (2013), entre 0 e 9 pontos não há triagem para EDM, entre 10 e 14 pontos há triagem para EDM leve, de 15 a 19 há EDM moderada e de 20 a 27 há triagem para EDM severa.

Escala Brasileira de Solidão UCLA (UCLA-BR). Desenvolvida por Russell, Peplau e Cutrona (1980) e validada para o contexto brasileiro por Barroso, Andrade, Midgett e Carvalho (2016), contém 20 afirmações, com respostas em escala tipo likert de quatro pontos, indo de 1 (nunca) a 4 (frequentemente). $\mathrm{O}$ instrumento mostrou boas qualidades psicométricas com consistência interna de 0,94 e boa capacidade de discriminação dos itens, avaliada por meio da Teoria de Resposta ao Item. O total de pontos é de até 60 , sendo: 0 a 22 pontos, indicativo de solidão mínima; 23 a 35 pontos, solidão leve; 36 a 47 pontos, solidão moderada; e 48 a 60 pontos, solidão intensa.

Escala de Percepção de Suporte Social (EPSS). Instrumento validado por Siqueira (2008), composto por 29 itens que avaliam suporte social prático e emocional. As respostas são apresentadas em escala likert de quatro pontos, indo de 1 (nunca) a 4 (sempre). O instrumento mostrou dois fatores que explicaram $47,33 \%$ da variância e alfa de Cronbach entre 0,91 e 0,92. A escala oferece três escores, um geral, um de suporte social prático e um emocional. A pontuação varia entre 32 e 144 pontos e não existe ponto de corte. Valores maiores indicam melhor rede de apoio social.

Questionário. Construído para este estudo com base nos fatores associados à depressão e a solidão identificados na literatura em Psicologia da Saúde. O questionário incluiu questões sociodemográficas, rotina de sono, alimentação, lazer, atividades físicas, participação em grupos da universidade e comunitários, doenças físicas e psiquiátricas, posse de animais de estimação, uso de medicação, área do curso de graduação, satisfação com o curso, relação com amigos, familiares, colegas de sala, sentimentos de tristeza e ansiedade. As perguntas eram dicotômicas ou em escala likert de cinco pontos.

\section{Coleta de Dados}

A coleta de dados ocorreu entre novembro de 2014 e maio de 2015. A aplicação dos instrumentos foi feita de forma coletiva, após autorização das coordenações de curso e docentes. O procedimento durou em média 30 minutos por aplicação. Paralelamente foi utilizada a plataforma virtual SurveyMonkey para coleta de dados. Nesse caso a coleta foi feita de forma individual, mediante divulgação em redes sociais.

\section{Análise de Dados}

Inicialmente procedeu-se com análises de frequência absoluta e relativa, medidas de tendência central e dispersão. Em seguida foram conduzidas análises de normalidade (teste Kolmogorov-Smirnov) para definição do tipo de teste inferencial a ser adotado. A análise de normalidade dos dados mostrou distribuição não-normal para solidão $(\mathrm{KS}=1,81$; $\mathrm{p}=0,003)$ e depressão $(\mathrm{KS}=2,91 ; \mathrm{p}<0,001)$, portanto, adotou-se estatística inferencial não-paramétrica. Para a investigação das relações entre as variáveis adotou-se a correlação de Spearman e para as diferenças entre subgrupos e o teste de Mann-Whitney para comparação entre dois grupos independentes e Kruskal-Wallis para três ou mais grupos independentes. Todas as análises inferenciais foram conduzidas com significância de 5\%, no programa SPSS, versão 17.0.

\section{Considerações Éticas}

Este estudo foi aprovado no Comitê de Ética em Pesquisa da UFTM (protocolo 2664) e todos os participantes assinaram o Termo de Consentimento Livre e Esclarecido. 


\section{RESULTADOS}

A maior parte dos participantes $(91,9 \%)$ considerava ter pelo menos um amigo ou mais e $73,1 \%$ mantinha contato com amigos de infância (Tabela 1). A maior parte dos estudantes indicou ter atividades de lazer dentro $(79,9 \%)$ e fora de casa $(86,6 \%)$. Mais da metade da amostra $(51,8 \%)$ praticava atividades físicas, não fumava $(90,6 \%)$ e consumia bebida alcóolica $(61,1 \%)$. A maior parte dos entrevistados (55\%) relatou fazer quatro ou mais refeições diárias entre segunda e sexta-feira e também nos finais de semana $(61,4 \%)$ e considerava sua alimentação como razoável, boa ou muito boa $(85,6 \%)$. A maioria dos entrevistados relatou dormir entre seis e sete horas de segunda a sexta-feira $(65,3 \%)$ e 8 horas ou mais nos finais de semana (62\%), além de considerarem a qualidade do sono como razoável, boa ou muito boa $(85,5 \%)$.

Tabela 1

Caracterização da Amostra, 2014-2015 (n= 574)

\begin{tabular}{|c|c|c|c|}
\hline Variável & Resposta & $\mathbf{n}$ & $\%$ \\
\hline \multirow{4}{*}{$\begin{array}{l}\text { Área de concentração do } \\
\text { curso }\end{array}$} & Ciências humanas & 103 & 18,0 \\
\hline & Ciências da saúde & 206 & 36,0 \\
\hline & Ciências exatas & 186 & 32,5 \\
\hline & Ciências naturais & 78 & 13,6 \\
\hline \multirow{2}{*}{ Sexo } & Feminino & 346 & 61,1 \\
\hline & Masculino & 220 & 38,9 \\
\hline \multirow{3}{*}{ Estado civil } & Solteiro & 534 & 93,4 \\
\hline & Casado & 35 & 6,1 \\
\hline & Separado/divorciado/viúvo & 03 & 0,5 \\
\hline \multirow{5}{*}{ Renda familiar } & Menos de 1 salário & 18 & 3,2 \\
\hline & 1 a 2 salários & 103 & 18,3 \\
\hline & 3 a 4 salários & 195 & 34,6 \\
\hline & 5 a 7 salários & 142 & 25,2 \\
\hline & Mais de 8 salários & 106 & 18,8 \\
\hline \multirow{4}{*}{ Horas sono em dias úteis } & 3 ou menos & 03 & 0,5 \\
\hline & 4 ou 5 & 127 & 22,3 \\
\hline & 6 ou 7 & 372 & 65,3 \\
\hline & 8 ou mais & 68 & 11,9 \\
\hline \multirow{4}{*}{$\begin{array}{l}\text { Horas sono em fins de } \\
\text { semana }\end{array}$} & 3 ou menos & 02 & 0,3 \\
\hline & 4 ou 5 & 26 & 4,5 \\
\hline & 6 ou 7 & 190 & 33,2 \\
\hline & 8 ou mais & 355 & 62,0 \\
\hline \multirow{5}{*}{ Autoavaliação do sono } & Muito ruim & 10 & 1,7 \\
\hline & Ruim & 73 & 12,7 \\
\hline & Razoável & 210 & 36,6 \\
\hline & Boa & 208 & 36,3 \\
\hline & Muito boa & 72 & 12,6 \\
\hline \multirow{2}{*}{ Acorda descansado } & Sim & 268 & 47,3 \\
\hline & Não & 299 & 52,7 \\
\hline \multirow{4}{*}{$\begin{array}{l}\mathrm{N}^{\circ} \text { de refeições em dias } \\
\text { úteis }\end{array}$} & 2 ou menos & 65 & 11,5 \\
\hline & 3 & 188 & 33,4 \\
\hline & 4 & 196 & 34,8 \\
\hline & 5 ou mais & 114 & 20,2 \\
\hline
\end{tabular}

Ao avaliar a solidão, observou-se que a maior parte dos estudantes apresentava nível mínimo (53,3\%), mas 2,4\% sentia solidão intensa (Tabela 2). Além disso, embora a maior parte dos estudantes não tenha sido triada para EDM (61,2\%), uma parcela expressiva recebeu essa classificação. Observou-se média de 82,91 pontos no suporte social (D.P. $+16,10$ ), sendo que $55,7 \%$ dos participantes ficou acima

As análises correlacionais mostraram correlação positiva moderada entre a classificação da solidão e depressão (rho $=0,42)$. Além disso, mostraram correlação negativa entre solidão e suporte social $($ rho $=-0,36)$ e entre depressão e suporte social $($ rho $=-0,25)($ Tabela 3$)$. desse valor. 
Tabela 1

Cont.

\begin{tabular}{|c|c|c|c|}
\hline Variável & Resposta & $\mathbf{n}$ & $\%$ \\
\hline \multirow{4}{*}{$\begin{array}{l}\mathrm{N}^{o} \text { de refeições nos fins de } \\
\text { semana }\end{array}$} & 2 ou menos & 43 & 7,5 \\
\hline & 3 & 177 & 31,3 \\
\hline & 4 & 225 & 39,5 \\
\hline & 5 ou mais & 125 & 21,9 \\
\hline \multirow{5}{*}{$\begin{array}{l}\text { Autoavaliação da } \\
\text { alimentação }\end{array}$} & Muito ruim & 14 & 2,4 \\
\hline & Ruim & 69 & 12,0 \\
\hline & Razoável & 263 & 45,9 \\
\hline & Boa & 198 & 34,6 \\
\hline & Muito boa & 29 & 5,1 \\
\hline \multirow{2}{*}{ Fuma } & Sim & 54 & 9,4 \\
\hline & Não & 518 & 90,6 \\
\hline \multirow{2}{*}{ Bebe } & Sim & 349 & 61,1 \\
\hline & Não & 222 & 38,9 \\
\hline \multirow{2}{*}{ Lazer em casa } & Sim & 448 & 79,9 \\
\hline & Não & 113 & 20,1 \\
\hline \multirow{2}{*}{ Lazer fora de casa } & Sim & 491 & 86,6 \\
\hline & Não & 76 & 13,4 \\
\hline \multirow{2}{*}{ Exercício físico } & Sim & 293 & 51,8 \\
\hline & Não & 273 & 48,2 \\
\hline \multirow{5}{*}{$\begin{array}{l}\text { Autoavaliação saúde } \\
\text { física }\end{array}$} & Muito ruim & 7 & 1,2 \\
\hline & Ruim & 62 & 10,8 \\
\hline & Razoável & 222 & 38,8 \\
\hline & Boa & 230 & 40,2 \\
\hline & Muito boa & 51 & 8,9 \\
\hline \multirow{5}{*}{$\begin{array}{l}\text { Autoavaliação saúde } \\
\text { mental }\end{array}$} & Muito ruim & 2 & 0,3 \\
\hline & Ruim & 33 & 5,8 \\
\hline & Razoável & 145 & 25,3 \\
\hline & Boa & 299 & 52,3 \\
\hline & Muito boa & 93 & 16,3 \\
\hline \multirow{5}{*}{ Tristeza últimos 15 dias } & Nenhum dia & 129 & 22,6 \\
\hline & 1 a 3 & 254 & 44,5 \\
\hline & 4 a 7 & 121 & 21,2 \\
\hline & 8 a 10 & 50 & 8,8 \\
\hline & 11 ou mais & 17 & 3,0 \\
\hline \multirow{5}{*}{ Ansiedade últimos 15 dias } & Nenhum dia & 39 & 6,8 \\
\hline & 1 a 3 & 198 & 34,6 \\
\hline & 4 a 7 & 168 & 29,4 \\
\hline & 8 a 10 & 92 & 16,1 \\
\hline & 11 ou mais & 75 & 13,1 \\
\hline \multirow{2}{*}{ Medicação } & Sim & 142 & 25,1 \\
\hline & Não & 423 & 74,9 \\
\hline \multirow{2}{*}{$\begin{array}{l}\text { Atividades grupo da } \\
\text { faculdade }\end{array}$} & Sim & 193 & 34,2 \\
\hline & Não & 372 & 65,8 \\
\hline \multirow{2}{*}{ Atividades voluntárias } & Sim & 69 & 12,2 \\
\hline & Não & 497 & 87,8 \\
\hline \multirow{2}{*}{$\begin{array}{l}\text { Atividades grupo fora da } \\
\text { faculdade }\end{array}$} & Sim & 150 & 26,5 \\
\hline & Não & 417 & 73,5 \\
\hline \multirow{2}{*}{ Tem amigo(s) } & Sim & 520 & 91,9 \\
\hline & Não & 46 & 8,1 \\
\hline \multirow{2}{*}{ Fez psicoterapia } & Sim & 122 & 21,6 \\
\hline & Não & 442 & 78,4 \\
\hline
\end{tabular}


Tabela 1

Cont.

\begin{tabular}{|c|c|c|c|}
\hline Variável & Resposta & $\mathbf{n}$ & $\%$ \\
\hline \multirow{2}{*}{ Animal de estimação } & Sim & 237 & 41,4 \\
\hline & Não & 336 & 58,6 \\
\hline \multirow{2}{*}{ Religião/crença religiosa } & Sim & 405 & 27,9 \\
\hline & Não & 157 & 72,1 \\
\hline \multirow{5}{*}{$\begin{array}{l}\text { Autoavaliação sobre seu } \\
\text { curso }\end{array}$} & Muito ruim & 15 & 2,6 \\
\hline & Ruim & 40 & 7,0 \\
\hline & Razoável & 149 & 26,2 \\
\hline & Bom & 239 & 42,0 \\
\hline & Muito bom & 126 & 22,1 \\
\hline \multirow{2}{*}{ Emprego } & Sim & 77 & 13,6 \\
\hline & Não & 489 & 86,4 \\
\hline \multirow{2}{*}{ Relacionamento afetivo } & Sim & 280 & 49,1 \\
\hline & Não & 290 & 50,9 \\
\hline \multirow{2}{*}{$\begin{array}{l}\text { Contato amigos de } \\
\text { infância }\end{array}$} & Sim & 418 & 73,1 \\
\hline & Não & 154 & 26,9 \\
\hline \multirow{5}{*}{$\begin{array}{l}\text { Relacionamento com } \\
\text { pessoas que mora }\end{array}$} & Muito ruim & 4 & 0,9 \\
\hline & Ruim & 8 & 1,7 \\
\hline & Razoável & 57 & 12,4 \\
\hline & Bom & 210 & 45,8 \\
\hline & Muito bom & 180 & 39,2 \\
\hline \multirow{5}{*}{$\begin{array}{l}\text { Relacionamento com } \\
\text { colegas de sala }\end{array}$} & Muito ruim & 3 & 0,5 \\
\hline & Ruim & 20 & 2,5 \\
\hline & Razoável & 102 & 17,8 \\
\hline & Bom & 319 & 55,7 \\
\hline & Muito bom & 129 & 22,5 \\
\hline \multirow{4}{*}{$\begin{array}{l}\text { Relacionamento com } \\
\text { o pai }\end{array}$} & Ruim & 108 & 19,4 \\
\hline & Regular & 182 & 32,6 \\
\hline & Bom & 239 & 42,8 \\
\hline & Ótimo & 29 & 5,2 \\
\hline \multirow{4}{*}{$\begin{array}{l}\text { Relacionamento com a } \\
\text { mãe }\end{array}$} & Ruim & 7 & 1,2 \\
\hline & Regular & 26 & 4,6 \\
\hline & Bom & 136 & 24,2 \\
\hline & Ótimo & 394 & 70,0 \\
\hline
\end{tabular}

Tabela 2

Prevalência de depressão, solidão e suporte social, 2014-2015 (n=574)

\begin{tabular}{|c|c|c|}
\hline Variável & $\mathbf{n}$ & $\%$ \\
\hline \multicolumn{3}{|l|}{ Triagem para Depressão } \\
\hline Não triado $(0-9)$ & 318 & 61,2 \\
\hline Leve (10-14) & 119 & 22,9 \\
\hline Moderada (15-19) & 63 & 12,1 \\
\hline Severa $(20-27)$ & 19 & 3,7 \\
\hline \multicolumn{3}{|l|}{ Intensidade Solidão } \\
\hline Mínima (0-22) & 306 & 53,3 \\
\hline Leve (23-35) & 205 & 35,7 \\
\hline Moderada (36-47) & 49 & 8,5 \\
\hline Severa (48-60) & 14 & 2,4 \\
\hline \multicolumn{3}{|l|}{ Suporte Social } \\
\hline Abaixo da média $(<82,7)$ & 254 & 44,3 \\
\hline Acima da média $(82,8>)$ & 320 & 55,7 \\
\hline
\end{tabular}


Tabela 3

Correlações significativas com solidão ou depressão

\begin{tabular}{|c|c|c|}
\hline & Classificação Solidão $^{1}$ & Triagem Depressão $^{1}$ \\
\hline Triagem positiva para depressão & $0,42 * *$ & - \\
\hline Suporte Social & $-0,36^{* *}$ & $-0,25^{* *}$ \\
\hline Área de concentração & $-0,14 * *$ & $-0,08$ \\
\hline Período do curso & $-0,12 *$ & $-0,14^{*}$ \\
\hline Sexo & $0,15^{* *}$ & $0,11 * *$ \\
\hline $\mathrm{N}^{o}$ de refeições nos dias úteis & $-0,09^{*}$ & $-0,11 *$ \\
\hline Autoavaliação da alimentação & $-0,13^{* *}$ & $-0,21 * *$ \\
\hline $\mathrm{N}^{o}$ de horas sono em dias úteis & $-0,10^{*}$ & $-0,14 * *$ \\
\hline Autoavaliação do sono & $-0,22 * *$ & $-0,29 * *$ \\
\hline Acorda descansado & $-0,16^{* *}$ & $0,34 * *$ \\
\hline Fumar & $-0,01$ & $0,11 *$ \\
\hline Doença crônica & 0,03 & $0,10^{*}$ \\
\hline Diagnóstico de transtorno psiquiátrico & $0,15^{* *}$ & $0,17 * *$ \\
\hline Uso contínuo de medicação & $0,09^{*}$ & $0,11 * *$ \\
\hline Autoavaliação da saúde física & $-0,20 * *$ & $-0,22 * *$ \\
\hline Autoavaliação da saúde mental & $-0,33 * *$ & $-0,45^{* *}$ \\
\hline Tristeza nos últimos 15 dias & $0,45^{* *}$ & $0,49 * *$ \\
\hline Ansiedade nos últimos 15 dias & $0,26 * *$ & $0,44 * *$ \\
\hline Já ter feito psicoterapia & $0,20 * *$ & $0,15^{* *}$ \\
\hline Autoavaliação do curso de graduação & $-0,17 * *$ & $-0,20 * *$ \\
\hline Renda familiar & $-0,10^{*}$ & $-0,14 * *$ \\
\hline Manter contato com amigos de infância & $-0,15^{* *}$ & $-0,09 *$ \\
\hline Relacionamento com as pessoas que mora & $-0,29 * *$ & $-0,21 * *$ \\
\hline Relacionamento com colegas & $-0,28 * *$ & $-0,15^{*}$ \\
\hline Atividade de lazer dentro de casa & $-0,09^{*}$ & $-0,09 *$ \\
\hline Atividade de lazer fora de casa & $-0,22 * *$ & $-0,16^{* *}$ \\
\hline Atividades de grupo ligadas a UFTM & $-0,06$ & $-0,04$ \\
\hline Atividades de grupo não ligadas a UFTM & $-0,09^{*}$ & $-0,11 * *$ \\
\hline Praticar esportes ou atividade física & $-0,15^{* *}$ & $-0,10^{*}$ \\
\hline Frequências com que pratica atividades físicas & $-0,23 * *$ & $-0,03$ \\
\hline Religião ou crença religiosa & $-0,16^{* *}$ & $-0,15^{* *}$ \\
\hline Considera ter um amigo ou mais & $-0,11^{* *}$ & $-0,08$ \\
\hline Frequência com que sai com os amigos & $-0,16^{* *}$ & $-0,14^{* *}$ \\
\hline Considerar-se uma pessoa com muitos amigos & $-0,24 * *$ & $-0,11 *$ \\
\hline Escala fazer novos amigos & $-0,37 * *$ & $-0,18 * *$ \\
\hline Escala manter amigos & $-0,25 * *$ & $-0,20 * *$ \\
\hline Anos de estudo do pai & $-0,09 *$ & $-0,00$ \\
\hline Relacionamento com o pai & $-0,12 * *$ & $-0,18 * *$ \\
\hline Relacionamento com a mãe & $-0,14^{* *}$ & $-0,14 * *$ \\
\hline Mãe estar viva & $0,25 * *$ & 0,04 \\
\hline
\end{tabular}

** $p<0,001 ; * p<0,05 ;{ }^{1}$ Correlação de Spearman

A triagem positiva para depressão foi maior entre as mulheres (rho $=0,11$ ), em pessoas com menor renda (rho $=-0,14)$, que estavam nos períodos iniciais da faculdade $($ rho $=-0,14)$ e avaliavam pior seu curso de graduação $(r h o=-0,20)$. As pessoas triadas para EDM faziam menos refeições durante os dias úteis (rho $=-0,11$ ), avaliavam pior a qualidade da sua alimentação $($ rho $=-0,21)$, dormiam menos horas nos dias úteis (rho $=-0,14)$, avaliavam pior sua qualidade do sono $($ rho $=-0,29)$ e acordavam cansados $($ rho $=0,34)$.

Observou-se correlação entre triagem para depressão e fumar $($ rho $=0,11)$, ter doença crônica $($ rho $=0,10)$, fazer uso contínuo de medicação (rho $=0,11)$, ter diagnóstico de transtorno psiquiátrico $(\mathrm{rho}=0,17)$, fazer uso contínuo de medicação $($ rho $=0,11)$, pior percepção sobre a saúde física $($ rho $=-0,22)$ e mental $($ rho $=-0,45)$, sentir-se triste (rho $=0,49)$ ou ansioso $($ rho $=0,44)$ nos 15 dias anteriores à entrevista, ter feito psicoterapia $($ rho $=0,15)$ e não ter religião ou crença religiosa $($ rho $=-0,15)$. A triagem para depressão também mostrou relação com pior percepção sobre o relacionamento com as pessoas com quem morava (rho $=-0,21)$ e com os colegas de sala $($ rho $=-0,15)$, percebiam ter poucos amigos $(\mathrm{rho}=-0,11)$, menos facilidade para fazer amigos $($ rho $=-0,18)$ e para mantê-los por longos períodos $($ rho $=-0,20)$ e classificavam de forma mais negativa seu relacionamento com seu pai $($ rho $=-0,18)$ e com a mãe $($ rho $=-0,14)$, Além disso, houve correlação entre a triagem para depressão e não ter atividades de lazer em casa (rho 
$=-0,09)$ e fora de casa (rho $=-0,16$ ), não participar das atividades de grupo da universidade (rho $=-0,11$ ), não praticar atividade física $($ rho $=-0,10)$ e sair menos com os amigos (rho $=-0,14)$.

Quanto à solidão, observou-se que as mulheres (rho = $0,15)$ e as pessoas com menor renda familiar $(\mathrm{rho}=-0,10)$ apresentaram níveis mais intensos de solidão. As pessoas mais solitárias avaliavam pior sua alimentação (rho $=-0,13$ ), dormiam menos nos dias úteis (rho $=-0,10$ ), avaliavam pior a qualidade do sono (rho $=-0,22$ ), sentiam-se menos descansados ao acordar $($ rho $=-0,16)$, tinham diagnóstico de transtorno psiquiátrico $(\mathrm{rho}=0,15)$, faziam uso contínuo de medicação (rho $=0,09)$, avaliavam pior sua saúde física (rho $=-0,20)$ e mental $($ rho $=-0,33)$, haviam se sentido tristes $($ rho $=0,45)$ ou ansiosos $($ rho $=0,26)$ nos 15 dias anteriores à entrevista e haviam feito psicoterapia $($ rho $=0,20)$.

Os resultados também mostraram maiores níveis de solidão nos estudantes dos períodos iniciais da graduação $($ rho $=-0,12)$ e classificavam pior seu curso (rho $=0,17)$. As pessoas mais solitárias relataram não ter atividade de lazer dentro $($ rho $=-0,09)$ e fora de casa $($ rho $=-0,22)$, não praticavam atividade física $($ rho $=-0,15)$ ou tinham menor frequência semanal de atividade física (rho $=-0,23$ ). As pessoas mais solitárias também indicaram não ter religião ou crença religiosa (rho $=-0,16$ ). Indicaram, ainda, não manter contato com amigos de infância (rho $=-0,15)$, não consideravam ter muito amigos $($ rho $=-0,24)$, indicaram dificuldade para fazer novos amigos (rho $=-0,37$ ), para manter amigos por longo tempo (rho $=-0,25)$, avaliavam pior seu relacionamento com o pai $($ rho $=-0,12)$ e com a mãe $($ rho $=-0,14)$, tinham mãe falecida $($ rho $=0,25)$ e saíam menos com amigos (rho $=-0,16$ ).

Ao avaliar a influência da área do curso sobre a depressão, observou-se que os estudantes dos cursos da área de Ciências Humanas (Geografia, História, Letras, Psicologia e Serviço Social) se mostraram mais deprimidos do que os respondentes de outras áreas $(\mathrm{KW}=15,36 ; \mathrm{p}=$ 0,002) (Tabela 4). Os estudantes que classificavam o próprio curso de pior maneira apresentaram mais triagem positiva para $\operatorname{EDM}(\mathrm{KW}=19,08 ; \mathrm{p}<0,001)$. Além disso, as mulheres

Tabela 4

Diferenças de grupo quanto à classificação da solidão e depressão

\begin{tabular}{|c|c|c|c|c|}
\hline & Média postos Solidão & Kruskal-Wallis & Média postos Depressão & Kruskal-Wallis \\
\hline \multicolumn{5}{|l|}{ Classificação da solidão } \\
\hline Mínima & - & - & 216,14 & $97,73 * *$ \\
\hline Leve & - & - & 290,21 & $97,73 * *$ \\
\hline Moderada & - & - & 378,14 & $97,73 * *$ \\
\hline Intensa & - & - & 399,50 & $97,73 * *$ \\
\hline \multicolumn{5}{|l|}{ Sexo } \\
\hline Feminino & 301,34 & $13,18^{* *}$ & 267,73 & $7,30 *$ \\
\hline Masculino & 255,44 & $13,18^{* *}$ & 236,01 & $7,30 *$ \\
\hline \multicolumn{5}{|l|}{ Área do curso } \\
\hline Ciências Humanas & 323,86 & $14,67^{*}$ & 287,20 & $15,36^{*}$ \\
\hline Ciências da Saúde & 298,03 & $14,67^{*}$ & 263,20 & $15,36^{*}$ \\
\hline Ciências Biológicas & 276,79 & $14,67^{*}$ & 228,56 & $15,36^{*}$ \\
\hline Ciências Exatas & 258,66 & $14,67^{*}$ & 281,01 & $15,36^{*}$ \\
\hline \multicolumn{5}{|l|}{ Autoavaliação do curso } \\
\hline Muito ruim & 365,23 & $19,68^{* *}$ & 336,79 & $22,00 * *$ \\
\hline Ruim & 349,08 & $19,68^{* *}$ & 311,70 & $22,00^{* *}$ \\
\hline Razoável & 300,50 & $19,68 * *$ & 281,61 & $22,00 * *$ \\
\hline Boa & 274,82 & $19,68^{* *}$ & 247,72 & $22,00 * *$ \\
\hline Muito boa & 256,09 & $19,68^{* *}$ & 229,25 & $22,00 * *$ \\
\hline \multicolumn{5}{|l|}{ Suporte social } \\
\hline Abaixo da média & 335,90 & $28345,50 * *$ & 286,76 & $27312,50^{* *}$ \\
\hline Acima da média & 249,08 & $28345,50 * *$ & 238,51 & $27312,50 * *$ \\
\hline \multicolumn{5}{|l|}{ Período do participante } \\
\hline 1 & 290,63 & $33,31 * *$ & 273,08 & $31,96 * *$ \\
\hline 2 & 367,82 & $33,31 * *$ & 341,13 & $31,96^{* *}$ \\
\hline 3 & 247,29 & $33,31 * *$ & 213,40 & $31,96^{* *}$ \\
\hline 4 & 282,83 & $33,31 * *$ & 264,24 & $31,96^{* *}$ \\
\hline 5 & 302,16 & $33,31 * *$ & 266,98 & $31,96^{* *}$ \\
\hline 6 & 276,43 & $33,31 * *$ & 237,17 & $31,96^{* *}$ \\
\hline 7 & 287,07 & $33,31 * *$ & 241,79 & $31,96^{* *}$ \\
\hline 8 & 231,10 & $33,31 * *$ & 227,38 & $31,96^{* *}$ \\
\hline 9 & 191,24 & $33,31 * *$ & 231,97 & $31,96^{* *}$ \\
\hline 10 & 286,80 & $33,31 * *$ & 199,36 & $31,96 * *$ \\
\hline
\end{tabular}

$* * p<0,001 ; * p<0,05$ 
mostraram mais triagem para depressão do que os homens (U $=7,30 ; \mathrm{p}=0,007)$, as pessoas mais solitárias apresentaram mais triagem para depressão $(\mathrm{KW}=97,73 ; \mathrm{p}<0,001)$ e as pessoas com suporte social abaixo da média apresentaram mais triagem para depressão $(U=27312,50 ; p<0,001)$.

Observou-se, também, que os participantes dos cursos da área de Ciências Humanas (Geografia, História, Letras, Psicologia e Serviço Social) mostraram maiores níveis de solidão do que os respondentes de outras áreas $(\mathrm{KW}=$ 14,67; $\mathrm{p}=0,002)$; e os estudantes que classificavam pior seu curso apresentaram maior nível de solidão ( $\mathrm{KW}=$ $19,68 ; \mathrm{p}=0,001)$. As mulheres também mostraram maior nível de solidão do que os homens $(\mathrm{U}=13,18 ; \mathrm{p}<0,001) \mathrm{e}$ as pessoas com suporte social abaixo da média da amostra apresentaram níveis mais altos de solidão do que as pessoas acima da média $(\mathrm{U}=28345,50 ; \mathrm{p}<0,001)$.

\section{DISCUSSÃO}

O presente estudo fez o levantamento do perfil dos estudantes universitários de uma instituição federal de Minas Gerais, incluindo seus níveis de solidão e depressão. Observou-se que os estudantes investigados apresentavam um perfil similar a outros estudantes da região sudeste, mas diferente do esperado para instituições federais brasileiras quanto a renda. Observou-se na amostra uma menor proporção de estudantes com renda até dois salários mínimos, o que difere do perfil nacional (FONAPRACE, 2019). Esse dado é relevante, uma vez que houve associação entre nível socioeconômico e nível de solidão, sugerindo que pessoas com menor renda se sentiram mais solitárias. Muitos eventos de socialização são pagos o que pode contribuir para excluir estudantes que não podem arcar com esses gastos, o que, em uma instituição de maior média de renda, pode aumentar o sentimento de exclusão percebida. Mas observou-se perfil semelhante de uso de álcool e tabaco com o identificado em outros estudos brasileiros (FONAPRACE, 2019; Rondina et al, 2018; Souza, 2017).

Ao observar os sintomas depressivos, percebeu-se que a porcentagem de pessoas triadas para EDM foi muito superior à observada para a população geral brasileira (IBGE, 2010), mas ficou próxima a observada em outros estudos com universitários (Furegato et al., 2010; Paula et al., 2014; Santos et al. 2015; Serra et al., 2015). No estudo de Souza (2017), as porcentagens de depressão foram ainda mais altas, tendo sido identificada triagem de EDM em 59,2\% dos estudantes.

Os sentimentos de solidão estiveram presentes em $46,7 \%$ dos estudantes da amostra do presente estudo, valor semelhante ao encontrado por Souza na mesma instituição em outro momento, que foi de 47\% (2017). Esse valor é o dobro do encontrado no perfil geral dos estudantes, que foi de 23,5\% (FONAPRACE, 2019). As mulheres se mostraram mais solitárias e apresentaram maior triagem para depressão, um resultado condizente com outros estudos sobre o transtorno já realizados (Kessler et al., 2010; OPS, 2018; WHO, 2012).

Os resultados também mostraram que os estudantes mais solitários apresentavam mais triagem para depressão, o que corrobora com os achados de outros estudos (Barroso, Baptista, \& Zanon, 2018; Souza, 2017), mostrando que a solidão é uma variável preditora da depressão. Os estudantes com menor suporte social também mostraram maior triagem para depressão, confirmando, mais uma vez, a relação entre solidão, baixo suporte social e risco aumentado para surgimento de transtorno depressivo (Ellwardt et al., 2013; Oliveira \& Silva, 2014; Rhodes, 2014; Russell et al., 2012; Tyler et al., 2011; WHO, 2012).

O impacto negativo da solidão também foi observado na saúde física e mental. Os resultados mostraram associação com a pior autopercepção sobre a saúde física e mental, a presença de doenças crônicas e transtornos psiquiátricos (Rhodes, 2014). Internacionalmente, Hackett et al. (2012) já haviam observado que pessoas mais solitárias apresentavam mais inflamações do que as menos solitárias. No mesmo estudo, as mulheres mais solitárias apresentaram mais estresse psicológico, índice de massa corporal e histórico de tabagismo. As relações observadas com hábitos alimentares, sono e percepção sobre a saúde com a depressão também já haviam sido identificadas em outras populações (Russell et al., 2012; Tyler et al., 2011).

Não foram encontradas relações significativas entre o uso de álcool, depressão ou solidão, mas houve relação entre fumar e ser triado para depressão, o que pode sugerir uma possível adoção de estratégia de enfrentamento problemática para lidar com um estado emocional negativo. Rondina et al. (2018) chamam a atenção para o fato de que os estudantes parecem ver nas drogas, em especial álcool, tabaco e maconha, uma espécie de mecanismo de enfrentamento para os problemas pessoais e relacionais pelos quais os universitários passam. A adoção de estratégias inadequadas pode afetar a saúde e o desempenho dos estudantes e os resultados podem clarear aspectos para serem abordados em intervenções preventivas e/ou terapêuticas que ajudem a escolher estratégias de enfrentamento mais funcionais.

Ao observarmos os resultados, percebe-se relações entre solidão, depressão e comprometimentos na vida dos estudantes. Observou-se relação da solidão e/ou depressão com a pior avaliação da própria saúde, diagnósticos anteriores de doenças físicas e psiquiátricas, sentir-se triste e ansioso, percepção negativa do relacionamento com colegas e familiares, percepção negativa do curso de graduação, dormir menos e com pior qualidade, fazer menos refeições por dia, mostrando claramente seu impacto sobre a qualidade de vida dos participantes. Guimarães (2014) observou que 
quanto maior a depressão, menor a qualidade de vida nos domínios psicológicos e ambiental, mas que também parece haver uma relação inversa com os domínios físico e social.

Ao pensar que a população avaliada no presente estudo foi de universitários, o impacto na vida assume, ainda, uma nova conotação, a do abandono dos planos futuros. Na pesquisa da FONAPRACE (2019) a associação entre vivenciar dificuldades emocionais e passar por reprovações e trancar ou abandonar a faculdade foi observada em uma parcela significativa de estudantes. Os resultados do presente trabalho mostraram uma relação significativa entre depressão, solidão e uma pior percepção do curso e do relacionamento com os colegas de sala, o que pode aumentar seu risco de abandonar a graduação, com consequências duradouras para a vida.

A análise das relações entre solidão, depressão e as áreas de concentração dos cursos demonstraram que os cursos de Ciências Humanas congregavam pessoas mais solitárias e deprimidas do que as demais áreas. Caso a associação fosse apenas com a solidão seria possível pensar que os cursos de humanas sensibilizassem mais para pensar sobre a qualidade dos vínculos estabelecidos. Contudo, houve também associação com a depressão, o que indica uma explicação mais negativa. Brandtner e Bardagi (2009) também observaram maior prevalência de depressão em estudantes da área de humanas, especialmente nos cursos de Letras e Psicologia. Esse resultado não é unânime, Rezende et al. (2018) avaliaram índices depressivos em universitários do interior do Rio de Janeiro, mas não encontraram diferenças significativas entre as áreas, no entanto sugerem que índices maiores de depressão nos estudantes de humanas podem estar relacionados a maior porcentagem de mulheres nesses cursos.
Estar nos períodos iniciais dos cursos de graduação também esteve relacionado com maiores níveis de solidão e maior triagem para depressão. Segundo Rhodes (2014), déficits nas competências sociais dos universitários ingressantes aumentam a chance do surgimento de sentimentos negativos, patologias físicas e transtornos psiquiátricos, o que poderia justificar esse aumento no sofrimento psíquico nos primeiros períodos. Outra possibilidade é o fato de que o primeiro ano é um período de adaptação à nova rotina, ao novo ambiente e as novas atividades cotidianas.

Mas os resultados também permitiram observar o potencial do suporte social e da adoção de algumas estratégias de enfrentamento para modular o impacto da depressão e da solidão. A atuação do suporte social como fator protetivo já havia sido identificada anteriormente, mostrando sua importância para o bem-estar e a qualidade de vida (Rhodes, 2014; Siqueira, 2008). As relações entre ter atividades de lazer dentro e fora de casa, ter amigos e sair com eles, ter uma crença religiosa e praticar atividade física e menores níveis de solidão e depressão indicam estratégias eficazes para promoção do bem-estar. Alguns desses fatores já haviam sido indicados por Baldissera e Bueno (2012) e Costa, Nakata e Morais (2015) como possíveis formas de intervenção para lidar com estados emocionais negativos.

É necessário, contudo, indicar algumas limitações desse trabalho. Foram incluídos estudantes de apenas uma instituição, não se utilizou amostra aleatória e os resultados foram baseados em medidas de autorrelato. Mas acredita-se que os resultados observados possam contribuir para suprir a lacuna de conhecimentos sobre a solidão da população brasileira.

\section{CONCLUSÃO}

A vida universitária é um período cheio de possibilidades, mas igualmente marcada pela necessidade de adaptação, cobranças e escolhas. Assim, embora rico, esse pode ser um período marcado por vivências de solidão e com risco aumentado para o surgimento de transtornos de humor, como a depressão. $\mathrm{O}$ desconhecimento sobre o estado emocional destes jovens adultos pode mascarar condições psicopatológicas e privá-los de acompanhamento, prejudicando sua qualidade de vida. Apesar dos índices de solidão observados não serem tão altos na população estudada, a frequência de triagem para EDM foi superior à média da população geral, indicando necessidade de pensar em propostas de intervenção. Tais propostas, individuais ou grupais poderão auxiliar no tratamento/prevenção dos sentimentos de solidão e sintomas depressivos entre os estudantes, especialmente se privilegiarem a construção de redes de suporte social entre os universitários ingressantes ou há menos tempo nos cursos de graduação. A elaboração de propostas que visem o bem-estar dos universitários é essencial, pois solidão e depressão acarretam consequências graves para a saúde, desempenho acadêmico e qualidade de vida. Os resultados também chamam a atenção para a necessidade de novos estudos sobre o estado emocional dos universitários, que incluam a avaliação da solidão em conjunto com a de outras emoções negativas, visando identificação precoce e tratamento aos que necessitarem. 


\section{REFERÊNCIAS}

Aquino, D. R. D., Cardoso, R. A., \& Pinho, L. D. (2019). Sintomas de depressão em universitários de medicina. Boletim-Academia Paulista de Psicologia, 39(96), 81-95.

Baldissera, V. D. A., \& Bueno, S. M. V. (2012). O lazer e a saúde mental das pessoas hipertensas: Convergência na educação para a saúde. Revista da Escola de Enfermagem da USP, 46(2), 380-387. doi:10.1590/S0080-62342012000200016

Bardagi, M. P., \& Hutz, C. S. (2011). Eventos estressores no contexto académico: Uma breve revisão da literatura brasileira. Interação em Psicologia, 15(1).

Barroso, S. M., Andrade, V. S., Midgett, A. H., \& Carvalho, R. G. N. (2016). Evidências de validade da Escala Brasileira de Solidão UCLA. Jornal Brasileiro de Psiquiatria, 65(1), 68-75. doi:10.1590/0047-2085000000105

Barroso, S. M., Andrade, V. S., Oliveira, N. R. (2016). Escala Brasileira de Solidão: Análises de Resposta ao Item e definição dos pontos de corte. Jornal Brasileiro de Psiquiatria, 65(1), 76-81. doi:10.1590/0047-2085000000106

Barroso, S. M., Baptista, M. N., \& Zanon, C. (2018). Solidão como variável preditora na depressão em adultos. Estudos Interdisciplinares em Psicologia, 9(3), 26-37. doi: 10.5433/2236-6407.2018v9n3suplp26

Bolsoni-Silva, A. T., \& Guerra, B. T. (2014). O impacto da depressão para as interações sociais de universitários. Estudos e Pesquisas em Psicologia, 14(2), 429-452.

Brandtner, M., \& Bardagi, M. (2009). Sintomatologia de depressão e ansiedade em estudantes de uma universidade privada do Rio Grande do Sul. Gerais: Revista Interinstitucional de Psicologia, 2(2), 81-91.

Cáceres, A. P. B., Cascaes, A. M., \& Büchele, F. (2010). Sintomas de disforia e depressão em estudantes de enfermagem. Cogitare Enfermagem, 15(4), 616-623. doi: 10.5380/ce.v15i4.20356

Cacioppo, J. T., Cacioppo, S. \& Boomsma, D. I. (2014). Evolutionary mechanisms for loneliness. Cognition and Emotion, 28(1), 3-21. doi:10.1080/02699931.2013.837379

Carlotto, R. C. (2013). Espiritualidade e sintomatologia depressiva em estudantes universitários brasileiros. Revista de Psicologia da UNESP, 12(2), 50-60.

Costa, F. M., Nakata, P. T., \& Morais, E. P. (2015). Estratégias desenvolvidas pelos idosos residentes na comunidade para morarem sozinhos. Texto \& Contexto - Enfermagem, 24(3), 818-825. doi:10.1590/0104-07072015002730014

Ellwardt, L., Aartsen, M., Deeg, D., \& Steverink, N. (2013). Does loneliness mediate the relation between social support and cognitive functioning in later life?. Social Science \& Medicine, 98, 116-124. doi:10.1016/j.socscimed.2013.09.002

Fórum Nacional de Pró-Reitores de Assuntos Comunitários e Estudantis. (2019). V Pesquisa do Perfil socioeconômico e cultural dos estudantes de graduação das instituições federais de ensino superior. Brasília, DF: FONAPRACE. Recuperado de http://www.andifes.org.br/wp-content/uploads/2019/05/VPesquisa-Nacional-de-Perfil-Socioecon $\%$ C3\%B4mico-eCultural-dos-as-Graduandos-as-das-IFES-2018.pdf

Furegato, A. R. F., Santos, J. L. F., \& Silva, E. C. (2010). Depressão entre estudantes de dois cursos de enfermagem: Autoavaliação da saúde e fatores associados. Revista Brasileira de Enfermagem, 63(4), 509-516. doi:10.1590/S003471672010000400002 .

Guimarães, M. F. (2014). Depressão, ansiedade, estresse e qualidade de vida de estudantes de universidades pública e privada (Dissertação de mestrado). Programa de Mestrado em Psicologia da Saúde, Universidade Metodista de São Paulo, SP, Brasil

Hackett, R. A., Hamer, M., Endrighi, R., Brydon, L., \& Steptoe, A. (2012). Loneliness and stress-related inflammatory and neuroendocrine responses in older men and women. Psychoneuroendocrinology, 37(11), 1801-1810. doi:10.1016/j. psyneuen.2012.03.016

Instituto Brasileiro de Geografia e Estatística. (2010). Censo demográfico 2010: Características da população e dos domicilios - Resultados do universo. Recuperado de http:// www.ibge.gov.br/english/estatistica/populacao/censo2010/ caracteristicas_da_populacao/resultados_do_universo.pdf

Instituto Brasileiro de Geografia e Estatística. (2014). Pesquisa Nacional de Saúde - 2013. Percepção do estado de saúde, estilos de vida e doenças crônicas. Rio de Janeiro: Instituto Brasileiro de Geografia de Estatística Recuperado de https:// biblioteca.ibge.gov.br/visualizacao/livros/liv94074.pdf

Kessler, R. C., Birnbaum, H., Shahly, V., Bromet, E., Hwang, I., McLaughlin, K. A., ... Stein, D. J. (2010). Age differences in the prevalence and co-morbidity of DSM-IV major depressive episodes: results from the WHO World Health Survey Initiative. Depression and Anxiety, 27(4), 351-364. doi:10.1002/da.20634

Liepins, M., \& Cline, T. (2011). The development of concepts of loneliness during the early years in school. School Psychology International, 35, 206-220. doi: 10.1177/0143034311404132

Marcus, M., Yasamy, M. T., van Ommeren, M., Chisholm, D., \& Saxena, S. (2012). Depression: A global public health concern. WHO Department of Mental Health and Substance Abuse, 1, 6-8. Recuperado de http://www.who.int/mental health/ management/depression/who_paper_depression_wfmh_2012. pdf

Molina, M. R. A. L., Wiener, C. D., Branco, J. C., Jansen, K., Souza, L. D. M., Tomasi, E., ... Pinheiro, R. T. (2012). Prevalência de depressão em usuários de unidades de atenção primária. Revista de Psiquiatria Clínica, 39(6), 194-197. doi:10.1590/ S0101-60832012000600003

Oliveira, A. P. S. V., \& Silva, M. M. (2014). Fatores que dificultam a perda de peso em mulheres obesas de graus I e II. Revista Psicologia e Saúde, 6(1), 74-82. Recuperado de: http://pepsic. bvsalud.org/pdf/rpsaude/v6n1/v6n1a10.pdf

Organização Pan-Americana de Saúde. (2018). Folha informativa: Depressão. Brasília, DF: Author. Recuperado de https://www. paho.org/bra/index.php?option=com_content\&view=article\& $\mathrm{id}=5635$ :folha-informativa-depressao $\&$ Itemid $=1095$

Paula, J. A., Borges, A. M. F. S., Bezerra, L. R. A., Parente, H. V., Paula, R. C. A., Wajnsztejn, R., ... Abreu, L. C. (2014). Prevalência e fatores associados à depressão em estudantes de medicina. Journal of Human Growth and Development, 24(3), 274-281. doi: 10.7322/jhdg.88911

Rezende, A. M., Vieira, E. M. S., Basili, J. O. V., Goulart, M., Ferreira, S. R., \& Barreto, M. A. M. (2018). Sintomas depressivos entre alunos de graduação: levantamento em instituição de ensino do interior do Rio de Janeiro. Cadernos UniFOA, 5(1), 60.

Rhodes, J. L. (2014). Loneliness: How superficial relationships, identity gaps, and social support contribute to feelings of loneliness at Pepperdine University. Pepperdine Journal of Communication Research, 2(3). Recuperado de: http:// digitalcommons.pepperdine.edu/pjcr/vol $2 /$ iss $1 / 3$

Rondina, R. C., Piovezzani, C. A. T., de Oliveira, D. C., \& Martins, R. A. (2018). Queixas psicológicas e consumo de drogas em universitários atendidos em núcleo de assistência. SMAD Revista Eletrônica Saúde Mental Álcool e Drogas, 14(2), 99-107.

Russell, D. W., Cutrona, C. E., McRae, C., \& Gomez, M. (2012). Is loneliness the same as being alone?. The Journal of Psychology, 146(1-2), 7-22. doi: 10.1080/00223980.2011.589414

Russell, D., Peplau, L. A., \& Cutrona, C. E. (1980). The revised UCLA Loneliness Scale: Concurrent and discriminant validity 
evidence. Journal of Personality and Social Psychology, 39(3), 472-480. doi:10.1037/0022-3514.39.3.472

Sakae, T. M., Padão, D. L., \& Jornada, L. K, (2010). Sintomas depressivos em estudantes da área da saúde em uma Universidade no sul de Santa Catarina - UNISUL. Revista da AMRIGS, 54(1), 38-43. Recuperado de: http://amrigs.org.br/ revista/54-01/11-473 sintomas_depressivos.pdf

Santos, I. S., Tavares, B. F., Munhoz, T. N., Almeida, L. S. P., Silva, N. T. B., Tams, B. D., ... Matijasevich, A. (2013). Sensibilidade e especificidade do Patient Health Questionnaire-9 (PHQ-9) entre adultos da população geral. Cadernos de Saúde Pública, 29(8), 1533-1543. doi:10.1590/0102-311X00144612

Serra, R. D., Dinato, S. L. M., \& Caseiro, M. M. (2015). Prevalence of depressive and anxiety symptoms in medical students in the city of Santos. Jornal Brasileiro de Psiquiatria, 64(3), 213-220. doi:10.1590/0047-2085000000081

Silveira, C., Norton, A., Brandão, I., \& Roma-Torres, A. (2011). Saúde mental em estudantes do ensino superior: Experiência da consulta de psiquiatria do Centro Hospitalar São João. Acta Médica Portuguesa, 24(S2), 247-256. Recuperado de https:// www.actamedicaportuguesa.com
Siqueira, M. M. M. (2008). Construção e validação da escala de percepção de suporte social. Psicologia em Estudo, 13(2), 381-388. doi:10.1590/S1413-73722008000200021

Souza, D. C. (2017). Condições emocionais de estudantes universitários: Stress, depressão, ansiedade, solidão e suporte social (Dissertação de mestrado). Universidade Federal do Triângulo Mineiro, Uberaba, MG, Brasil. Recuperado de http:// bdtd.uftm.edu.br/handle/tede/507

Tabalipa, F. O., Souza, M. F., Pfützenreuter, G., Lima, V. C., Traebert, E., \& Traebert, J. (2015). Prevalence of anxiety and depression among medical students. Revista Brasileira de Educação Médica, 39(3), 388-394. doi:10.1590/1981$52712015 \mathrm{v} 39 \mathrm{n} 3 \mathrm{e} 02662014$

Tyler, J., Hawkley, L. C., Thisted, R. A., \& Cacioppo, J. T. (2011). A marginal structural model analysis for loneliness: Implications for intervention trials and clinical practice. Journal of Consulting and Clinical Psychology, 79(2), 225-235. doi: $10.1037 / \mathrm{a} 0022610$

World Health Organization. (2012). The World Health Report 2012. Depression. Genebra: Author. Recuperado de http://www.who. int/topics/depression/en/. 